\title{
Effect of recombinant human prourokinase on thrombolysis in a rabbit model of thromboembolic stroke
}

\author{
CHUNHUA HAO $^{1^{*}}$, WENXIA DING ${ }^{2 *}$, XIANGWEI XU ${ }^{1}$, QIAN SUN $^{2}$, \\ XINXIN LI ${ }^{2}$, WEITING WANG ${ }^{1}$, ZHUANYOU ZHAO ${ }^{1}$ and LIDA TANG ${ }^{1}$
}

\author{
${ }^{1}$ State Key Laboratory of Pharmacokinetics and Pharmacodynamics, Tianjin Institute of Pharmaceutical Research, \\ Tianjin 300010; ${ }^{2}$ Institute of Pharmacology and Toxicology, Tasly Pharmaceutical Co., Ltd., Tianjin 300412, P.R. China
}

Received July 26, 2017; Accepted October 10, 2017

DOI: 10.3892/br.2017.1013

\begin{abstract}
The aim of the present study was to investigate the efficacy of recombinant human prourokinase (rhPro-UK) on thromboembolic stroke in rabbits. A total of 210 rabbits were used in experiments. The 180 thromboembolic stroke rabbits were divided into three therapeutic time windows with six groups in each time window $(\mathrm{n}=10)$. The model group was administered saline, the reagent groups were administered rhPro-UK $\left(2.5 \mathrm{x}, 5 \mathrm{x}\right.$ and $\left.10 \times 10^{4} \mathrm{U} / \mathrm{kg}\right)$, and the positive control groups were administered $5 \times 10^{4}$ urokinase (UK) $\mathrm{U} / \mathrm{kg}$ and $4.5 \mathrm{mg} / \mathrm{kg}$ recombinant human tissue plasminogen activator via intravenous infusion at $3,4.5$ and $6 \mathrm{~h}$ after embolism. The remaining 30 rats (that had not undergone occlusion by autologous blood clots) served as a sham group and were administered saline. The radioactive intensity was detected using a medical gamma counter before and after the administration of the drug for 15, 30, 45, 60, 75, 90, 105 and $120 \mathrm{~min}$. At $24 \mathrm{~h}$ after treatment, the brain samples were coronally sliced into $5 \mathrm{~mm}$ sections and hemorrhage was estimated used a semiquantitative method by counting the number of section faces with hemorrhaging. The plasma was collected for prothrombin time, activated partial thromboplastin time, fibrinogen and thrombin time tests using a solidification method with a blood coagulation factor analyzer. In addition, $\alpha_{2}$-antiplasmin $\left(\alpha_{2}\right.$-AP) was evaluated using ELISA methods using a RT-6100 microplate reader. At the $3 \mathrm{~h}$ time point, the thrombolysis rate of rhPro-UK $\left(2.5 \mathrm{x}, 5 \mathrm{x}\right.$ and $\left.10 \times 10^{4} \mathrm{U} / \mathrm{kg}\right)$
\end{abstract}

Correspondence to: Professor Weiting Wang or Dr Lida Tang, State Key Laboratory of Pharmacokinetics and Pharmacodynamics, Tianjin Institute of Pharmaceutical Research, 308 Huiren Road, Binhai Science and Technology Park, Binhai High-Tech Zone, Tianjin 300010, P.R. China

E-mail:wangwt@tjipr.com

E-mail: tangld@tjipr.com

*Contributed equally

Key words: technetium, recombinant human prourokinase, rabbit, thromboembolic stroke, recombinant human tissue plasminogen activator was $21.5 \%(\mathrm{P}<0.05), 36.8 \%(\mathrm{P}<0.001)$ and $55.0 \%(\mathrm{P}<0.001)$, respectively together with patency rates of $10 \%(\mathrm{P}>0.05)$, $40 \%(\mathrm{P}<0.05)$ and $70 \%(\mathrm{P}<0.001)$. Furthermore, $\alpha_{2}$-AP levels were reduced by $5.3 \%(\mathrm{P}>0.05), 5.3 \%(\mathrm{P}>0.05)$ and $18.1 \%$ $(\mathrm{P}<0.05)$. At the $4.5 \mathrm{~h}$ time point, the thrombolysis rate was $18.8 \%(\mathrm{P}<0.05), 29.9 \%(\mathrm{P}<0.01)$ and $49.0 \%(\mathrm{P}<0.001)$ together with patency rates of $10 \%(\mathrm{P}>0.05), 30 \%(\mathrm{P}<0.05)$ and $50 \%$ $(\mathrm{P}<0.01)$, and $\alpha_{2}$-AP levels were reduced by $2.4 \%(\mathrm{P}>0.05)$, $6.5 \%(\mathrm{P}>0.05)$ and $17.8 \%(\mathrm{P}<0.05)$. At the $6 \mathrm{~h}$ time point, the thrombolysis rate was $14.7 \%(\mathrm{P}<0.05), 24.1 \%(\mathrm{P}<0.01)$ and $35.7 \%(\mathrm{P}<0.001)$ together with patency rates of $20 \%$ $(\mathrm{P}>0.05), 30 \%(\mathrm{P}<0.05)$ and $40 \%(\mathrm{P}<0.01)$, and $\alpha_{2}$-AP levels were reduced by $5.7 \%(\mathrm{P}>0.05), 12.7 \%(\mathrm{P}>0.05)$ and $22.2 \%$ $(P<0.01)$. No significant differences $(P>0.05)$ were identified between rhPro-UK $\left(2.5 \mathrm{x}, 5 \mathrm{x}\right.$ and $\left.10 \mathrm{x} 10^{4} \mathrm{U} / \mathrm{kg}\right)$ and the model group regarding hemorrhage type, size and blood coagulation factors at the different time points. Thus, rhPro-UK promoted thrombolysis and recanalization (patency rate), with reduced risk of cerebral hemorrhage, and thus exerted protective effects on cerebral ischemia rabbits.

\section{Introduction}

Urokinase (UK), also termed UK-type plasminogen activator (uPA), is a type of serine protease present in humans and other animals, used clinically as a thrombolytic agent in the treatment of severe or massive deep venous thrombosis (DVT), pulmonary embolism, myocardial infarction, and occluded intravenous (IV) or dialysis cannulas. However, UK is not particularly selective for clot-bound plasminogen (it binds almost equally to freely circulating plasminogen and clot-bound plasminogen), and causes significant fibrinogenolysis and clot fibrinolysis. To the best of our knowledge, prourokinase (Pro-UK; also termed single-chain UK-type PA, single-chain pro-UK, scu-PA, pro-UK, pro u-PA and PUK) has only been evaluated in stroke by a single study (1). Pro-UK is a zymogen with little fibrin affinity, but has an equivalent fibrin specificity to tissue PA (tPA) (2). Intra-arterial local rpro-UK infusion has previously been associated with superior recanalization in acute thrombotic/thromboembolic stroke when compared with a placebo (3). The safety and efficacy of the thrombolytic agent, pro-UK, in the treatment of DVT of the lower limbs have been investigated in an open, uncontrolled, pilot study (4). The results 
of this pilot study indicated that pro-UK was thrombolytic in DVT and that it may be administered simultaneously with a conventional heparin treatment. Recombinant human (rh) Pro-UK, is a novel type of thrombolytic, which preferentially activates plasminogen on the fibrin surface and induces fibrin-selective clot lysis. It has the advantages of more potent efficacy and less adverse reactions in comparison with other thrombolytics (5). Advantages of thrombolytic therapy using rhPro-UK for patients with acute myocardial infarction include its reliable curative effect and high safety (6). The aim of the present study was to investigate the effects of rhPro-UK in rabbit models of thromboembolic stroke at 3, 4.5 and $6 \mathrm{~h}$ therapeutic time windows, particularly regarding its effects on thrombolysis rate, patency rate (recanalization) and intracerebral hemorrhage.

\section{Materials and methods}

Animals. Adult male and female rabbits, weighing $2.0-3.0 \mathrm{~kg}$ [SCXK(JING)2014-0003] were obtained from Longan Experimental Animal Breeding Center(Beijing, China). The present study was performed in strict accordance with the recommendations in the Guide for the Care and Use of Laboratory Animals of the Tianjin Institute of Pharmaceutical Research (Tianjin, China).

Drugs, reagents and devices. rhPro-UK (batch no. 201400401) was obtained from Shanghai Tasly Pharmaceutical Co., Ltd. (Shanghai, China). Recombinant human tissue plasminogen activator (rt-PA; batch no. 403437) was obtained from Boehringer Ingelheim (Ingelheim am Rhein, Germany). UK (batch no. 041603023) was obtained from Biochemical Pharmaceutical Co., Ltd. (Tianjin, China).

The rabbit $\alpha_{2}$-AP ELISA kit (batch no. 201606) was provided by Bio-Swamp Co., Ltd. (Wuhan, China), and assay kits for activated partial thromboplastin time (APTT; batch no. 021603A), prothrombin time (PT; batch no. 011504A), thrombin time (TT; batch no. 031601D) and fibrinogen (FIB; batch no. 041603A) were obtained from MD Pacific (Tianjin, China ) Biotechnology Co., Ltd. (Tianjin, China), technetium $\left({ }^{99} \mathrm{Tcm}\right)$ sodium was obtained from Atomic High Tech Isotope Pharmaceutical Co., Ltd. (Tianjin, China).

A JC1000-PC Medical Gamma Counter was obtained from Kaipu Electromechanical Co., Ltd.(Xi'an, China), RT-6100 microplate reader was purchased from Rayto Life Science Co., Ltd., (Guangzhou, China) and a PARBER blood coagulation factor analyzer was obtained from Beijing SHIDI Scientific Instrument Company (Beijing, China).

\section{Establishing the experimental embolism model}

Embolus preparation. Embolism was established as previously described $(7,8)$ with some modification. Briefly, labeled mixture was obtained from $0.5 \mathrm{ml}$ eluted radioactive sodium (radioactive intensity, 92.5 MBq/ml) with $30 \mu 1$ stannous chloride $(5 \mathrm{mg} / \mathrm{ml})$. Of this, $20 \mu \mathrm{l}$ was added into $1 \mathrm{ml}$ rabbit anticoagulant blood and incubated for $30 \mathrm{~min}$ at $37^{\circ} \mathrm{C}$. Mixture $(50 \mu \mathrm{l})$ with an equal volume of $\mathrm{CaCl}_{2}(0.5 \mathrm{M})$ and bovine thrombin $(50 \mathrm{IU} / \mathrm{ml})$ were added into the rabbit autologous blood and a PE90 pipe (inner diameter, $0.86 \mathrm{~mm}$; outer diameter, $1.27 \mathrm{~mm}$ ) was used to collect a clot sample; the clot was solidified at $37^{\circ} \mathrm{C}$ for $2 \mathrm{~h}$ and sliced to $10 \mathrm{~mm}$. The radioactivity of the thrombus was evaluated using a JC1000-PC Medical Gamma Counter following three washes with normal saline (5 $\mathrm{min} /$ wash).

Establishing the embolism model. The rabbits were anesthetized using $20 \%$ urethane $(1 \mathrm{~g} / \mathrm{kg})$ and fixed on the operating table. The cervical midline skin was incised and the right carotid artery, internal carotid artery (ICA) and external carotid artery (ECA) were separated. Following ligation and transection of the ECA, the modified PE90 (reducing the optical density to $0.4 \mathrm{~mm}$ at one end) with blood clot samples was injected with a $2 \mathrm{ml}$ syringe via the ECA into the ICA. The radioactive intensity of the forelimb cortex $(1.0 \mathrm{~mm}$ posterior and $5.5 \mathrm{~mm}$ lateral to the bregma) was detected using a JC1000-PC Medical Gamma Counter prior to and following embolus injection (9-11). When the radiation intensity was $>2$ times greater than the background signal, the model preparation for thromboembolism was considered successful (8). All rabbits were resuscitated following completion of the thrombolysis assay.

Grouping. The animals with thromboembolic stroke were randomly divided into 6 groups as follows: Model group (saline solution), the rhPro-UK $\left(2.5 \mathrm{x}, 5 \mathrm{x}\right.$ and $\left.10 \times 10^{4} \mathrm{U} / \mathrm{kg}\right)$ groups and the positive groups $\left(5 \times 10^{4} \mathrm{U} / \mathrm{kg} \mathrm{UK}\right.$ and $\left.4.5 \mathrm{mg} / \mathrm{kg} \mathrm{rt}-\mathrm{PA}\right)$. In addition, the rabbits in the sham group without occlusion by autologous blood clots were administered saline solution. A total of 10 rabbits in each group were treated at 3, 4.5 and $6 \mathrm{~h}$ after occlusion via IV infusion.

Thrombolysis assay. The radioactive intensity was detected using a medical gamma counter before and after drug administration for 15, 30, 45, 60, 75, 90, 105 and $120 \mathrm{~min}$. A thrombolysis rate $>50 \%$ was considered as the patency rate (12) and calculated as follows: Thrombolysis rate $(\%)=\left[\left(\mathrm{n}_{0} \mathrm{x}\right.\right.$ $\left.\mathrm{k}-\mathrm{nt}) /\left(\mathrm{n}_{0} \times \mathrm{k}\right)\right] \mathrm{x} 100 \%$ where $\mathrm{k}=\mathrm{e}^{-0.6931 \times(\mathrm{t} / 6.02)}, \mathrm{n}_{0}$ is the radioactive intensity before administration, $\mathrm{n}_{\mathrm{t}}$ is the radioactive intensity after different drug administration times and $t$ is the time after administration.

Intracerebral hemorrhage assay. The animals were sacrificed $24 \mathrm{~h}$ after treatment under anesthesia with $20 \%$ urethane $(1 \mathrm{~g} / \mathrm{kg})$. Brains were removed subsequent to perfusion and coronally sliced into $5 \mathrm{~mm}$ sections. The hemorrhage was estimated used a semi-quantitative method by counting the number of sections where hemorrhage was present (13-16). Each brain slice has two 'faces' and the score counting criteria were 1 for a hemorrhage on 1 'face'and 2 for a hemorrhage on 2 'faces', then the total bleeding score was calculated. Three types of hemorrhage were identified as follows: i) Hemorrhagic infarction or red speckling of an area, usually surrounded by soft infarcted tissue; ii) punctate hemorrhages or isolated small red marks within the tissue; and iii) parenchymatous intracerebral hemorrhages, a large homogeneous mass of blood within the tissue.

Blood coagulation factor determination. Blood was collected by heart puncture and anticoagulated with $3.8 \%$ sodium citrate and the plasma was obtained by centrifugation $\left(4^{\circ} \mathrm{C}\right.$, $1,000 \mathrm{x} g$ for $10 \mathrm{~min})$. PT, APTT, TT and FIB were evaluated using a solidification method, according to manufacturer's 
Table I. Effect of rhPro-UK on patency rate in thromboembolic rabbit models (means \pm standard error; $n=10$ ).

\begin{tabular}{|c|c|c|c|}
\hline $\begin{array}{l}\text { Therapeutic time } \\
\text { window (h) }\end{array}$ & Group & $\begin{array}{c}\text { Dose } \\
\left(\mathrm{x} 10^{4} \mathrm{U} / \mathrm{kg}\right)\end{array}$ & $\begin{array}{c}\text { Patency rate } \\
(\%)\end{array}$ \\
\hline \multirow{8}{*}{3} & Sham & - & - \\
\hline & Model & - & 0 \\
\hline & rhPro-UK & 2.5 & 10 \\
\hline & rhPro-UK & 5 & $40^{\mathrm{a}, \mathrm{d}}$ \\
\hline & rhPro-UK & 10 & $70^{c}$ \\
\hline & UK & 5 & 20 \\
\hline & rt-PA & $4.5 \mathrm{mg} / \mathrm{kg}$ & $40^{\mathrm{a}}$ \\
\hline & Sham & - & - \\
\hline \multirow{4}{*}{4.5} & Model & - & 0 \\
\hline & rhPro-UK & 2.5 & 10 \\
\hline & rhPro-UK & 5 & $30^{\mathrm{a}, \mathrm{d}}$ \\
\hline & rhPro-UK & 10 & $50^{\mathrm{b}}$ \\
\hline \multirow{9}{*}{6} & UK & 5 & 20 \\
\hline & rt-PA & $4.5 \mathrm{mg} / \mathrm{kg}$ & $30^{\mathrm{a}}$ \\
\hline & Sham & - & - \\
\hline & Model & - & 0 \\
\hline & rhPro-UK & 2.5 & 20 \\
\hline & rhPro-UK & 5 & $30^{\mathrm{a}, \mathrm{d}}$ \\
\hline & rhPro-UK & 10 & $40^{\mathrm{b}}$ \\
\hline & UK & 5 & 0 \\
\hline & rt-PA & $4.5 \mathrm{mg} / \mathrm{kg}$ & 20 \\
\hline
\end{tabular}

${ }^{\mathrm{a}} \mathrm{P}<0.05 ;{ }^{\mathrm{b}} \mathrm{P}<0.01 ;{ }^{\mathrm{c}} \mathrm{P}<0.001$ vs. model group. ${ }^{\mathrm{d}} \mathrm{P}>0.05,5 \times 10^{4} \mathrm{U} / \mathrm{kg}$ rhPro-UK vs. UK. rhPro-UK, recombinant human prourokinase; UK, urokinase; rt-PA, recombinant human tissue plasminogen activator.

instructions of the PT, APTT, TT and FIB assay kits, with the PARBER Blood Coagulation Factor Analyzer. Levels of $\alpha_{2}$-AP were also measured via ELISA with the RT-6100 microplate reader.

Statistical analysis. Values are presented as the mean \pm standard error of the mean, normal distribution data were analyzed using one-way analysis of variance and non-normal data were evaluated using a nonparametric test, the Kruskal Wallis test. The counting data are expressed as ratios (\%) and using the $\chi^{2}$ test $\mathrm{P}<0.05$ was considered to indicate a statistically significant difference.

\section{Results}

Effect of rhPro-UK on thrombolysis. At the $3 \mathrm{~h}$ therapeutic time window, the patency rate of $\operatorname{rhPro}-\mathrm{UK}\left(2.5 \mathrm{x}, 5 \mathrm{x}\right.$ and $\left.10 \times 10^{4}\right)$ was $10 \%(\mathrm{P}>0.05), 40 \%(\mathrm{P}<0.05)$ and $70 \%(\mathrm{P}<0.001)$, and the thrombolysis rate reached $21.5 \%(\mathrm{P}<0.05), 36.8 \%(\mathrm{P}<0.001)$ and $55.0 \%(\mathrm{P}<0.001)$, respectively. At $4.5 \mathrm{~h}$ post-embolism, the patency rate was increased to $10 \%(\mathrm{P}>0.05), 30 \%(\mathrm{P}<0.05)$ and $50 \%(\mathrm{P}<0.01)$, and the thrombolysis rate reached $18.8 \%$ $(\mathrm{P}<0.05), 29.9 \%(\mathrm{P}<0.01)$ and $49.0 \%(\mathrm{P}<0.001)$, respectively. At $6 \mathrm{~h}$ post-embolism, the patency rate was increased to $20 \%$
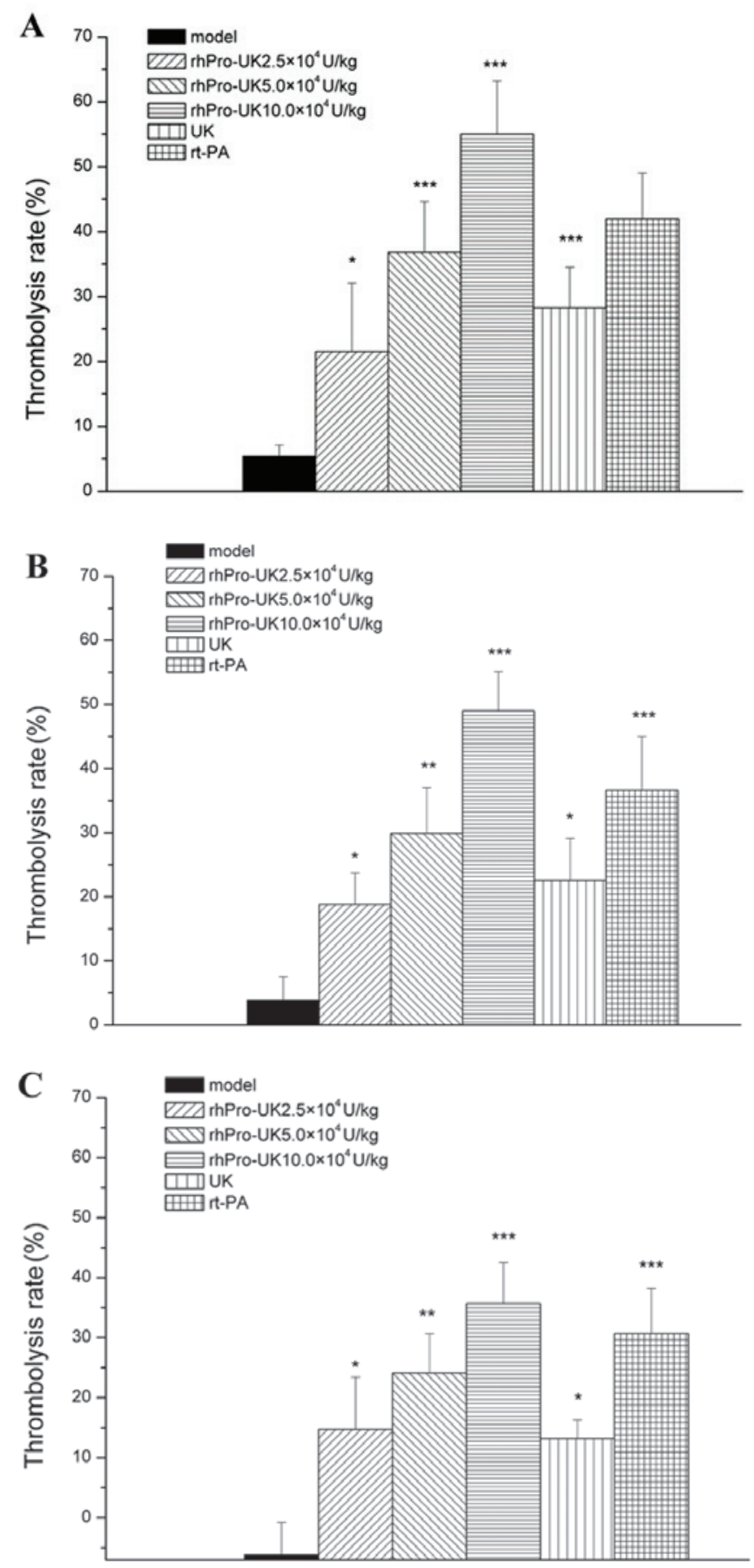

Figure 1. Effect of rhPro-UK on thrombolysis in thromboembolic rabbits. Thrombolysis rate was measured at: (A) 3, (B) 4.5 and (C) $6 \mathrm{~h}$ therapeutic time windows. Data are presented as means \pm standard error $(\mathrm{n}=10)$. ${ }^{*} \mathrm{P}<0.05$, ${ }^{* *} \mathrm{P}<0.01$ and ${ }^{* * *} \mathrm{P}<0.001$ vs. model group. rhPro-UK5 $\times 10^{4} \mathrm{U} / \mathrm{kg}$ compared with UK, P>0.05. rhPro-UK, recombinant human prourokinase; UK, urokinase; rt-PA, recombinant human tissue plasminogen activator.

$(\mathrm{P}>0.05), 30 \%(\mathrm{P}<0.05)$ and $40 \%(\mathrm{P}<0.01)$, and the thrombolysis rate reached $14.7 \%(\mathrm{P}<0.05), 24.1 \%(\mathrm{P}<0.01)$ and $35.7 \%(\mathrm{P}<0.001)$.

At the 3, 4.5 and $6 \mathrm{~h}$ therapeutic time windows, the patency rate of $5 \times 10^{4} \mathrm{U} / \mathrm{kg} \mathrm{UK}$ was $20 \%(\mathrm{P}>0.05), 20 \%(\mathrm{P}>0.05)$ and $0 \%(\mathrm{P}>0.05)$, respectively and the patency rate of $4.5 \mathrm{mg} / \mathrm{kg}$ rt-PA was $40 \%(\mathrm{P}<0.05), 30 \%(\mathrm{P}<0.05)$ and $20 \%(\mathrm{P}>0.05)$. rhProUK $\left(5 \times 10^{4} \mathrm{U} / \mathrm{kg}\right)$ marginally increased the thrombolysis 
Table II. Effect of rhPro-UK on thrombolysis rate (\%) and thrombolysis time in thromboembolic rabbit models at the $3 \mathrm{~h}$ therapeutic window (means \pm standard error; $\mathrm{n}=10$ ).

\begin{tabular}{|c|c|c|c|c|c|c|c|c|c|c|}
\hline \multirow[b]{2}{*}{ Group } & \multirow{2}{*}{$\begin{array}{l}\text { Dose } \\
\left(\mathrm{x} 10^{4}\right. \\
\mathrm{U} / \mathrm{kg})\end{array}$} & \multirow{2}{*}{$\begin{array}{l}\text { Pre- } \\
\text { adminis- } \\
\text { tration }\end{array}$} & \multicolumn{8}{|c|}{ Time after administration (min) } \\
\hline & & & 15 & 30 & 45 & 60 & 75 & 90 & 105 & 120 \\
\hline Model & - & 0 & $3.0 \pm 1.7$ & $3.4 \pm 2.9$ & $3.1 \pm 2.9$ & $5.5 \pm 4.5$ & $2.0 \pm 2.5$ & $4.3 \pm 3.7$ & $5.5 \pm 4.0$ & $5.4 \pm 1.7$ \\
\hline hPro-UK & 2.5 & 0 & $0.2 \pm 4.2$ & $5.2 \pm 4.3$ & $6.2 \pm 3.8$ & $8.0 \pm 5.1$ & $15.2 \pm 4.8^{\mathrm{a}}$ & $17.6 \pm 7.4$ & $23.6 \pm 6^{\mathrm{a}}$ & $21.5 \pm 10.6$ \\
\hline rhPro-UK & 5 & 0 & $10.3 \pm 2.6^{\mathrm{d}}$ & $7.6 \pm 2.4^{\mathrm{d}}$ & $9.2 \pm 3.1^{\mathrm{d}}$ & $23.5 \pm 4.5^{\mathrm{a}, \mathrm{d}}$ & $25.9 \pm 4.3^{\mathrm{c}, \mathrm{d}}$ & $33.4 \pm 5.8^{\mathrm{b}, \mathrm{d}}$ & $32.4 \pm 6.1^{\mathrm{b}, \mathrm{d}}$ & $36.8 \pm 7.8^{\mathrm{c}, \mathrm{c}}$ \\
\hline rhPro-UK & 10 & 0 & $17.4 \pm 2.7^{b}$ & $28.2 \pm 5.1^{\mathrm{c}}$ & $38.5 \pm 6.5^{\mathrm{c}}$ & $39.3 \pm 8.5^{\mathrm{c}}$ & $42.7 \pm 7.8^{c}$ & $49.2 \pm 9.7^{c}$ & $53.3 \pm 8.8^{c}$ & $55.0 \pm 8.2^{\mathrm{c}}$ \\
\hline UK & 5 & 0 & $8.8 \pm 4.8$ & $9.7 \pm 4.6$ & $13.6 \pm 2.8^{\mathrm{a}}$ & $16.8 \pm 3.8$ & $16.8 \pm 3.4^{\mathrm{a}}$ & $22.1 \pm 7.1^{\mathrm{a}}$ & $22.2 \pm 7.2$ & $28.3 \pm 6.2^{\mathrm{c}}$ \\
\hline rt-PA & $\begin{array}{c}4.5 \\
\mathrm{mg} / \mathrm{kg}\end{array}$ & 0 & $9.5 \pm 3.4$ & $16.1 \pm 4.4^{\mathrm{a}}$ & $19.1 \pm 6.9^{\mathrm{a}}$ & $23.8 \pm 7.6^{\mathrm{a}}$ & $30.8 \pm 9.2^{c}$ & $33.4 \pm 8.5^{\mathrm{b}}$ & $35.6 \pm 8.1^{\mathrm{b}}$ & $42.0 \pm 7.0^{c}$ \\
\hline
\end{tabular}

${ }^{\mathrm{a}} \mathrm{P}<0.05,{ }^{\mathrm{b}} \mathrm{P}<0.01$ and ${ }^{\mathrm{c}} \mathrm{P}<0.001$ vs. model group; ${ }^{\mathrm{D}}>0.05,5 \times 10^{4} \mathrm{U} / \mathrm{kg}$ rhPro-UK vs. UK. rhPro-UK, recombinant human prourokinase; UK, urokinase; rt-PA, recombinant human tissue plasminogen activator.

Table III. Effect of rhPro-UK on thrombolysis rate (\%) and thrombolysis time in thromboembolic rabbit models at the $4.5 \mathrm{~h}$ therapeutic window (means \pm standard error; $\mathrm{n}=10$ ).

\begin{tabular}{|c|c|c|c|c|c|c|c|c|c|c|}
\hline \multirow[b]{2}{*}{ Group } & \multirow{2}{*}{$\begin{array}{l}\text { Dose } \\
\text { (x10 } \\
\mathrm{U} / \mathrm{kg})\end{array}$} & \multirow{2}{*}{$\begin{array}{l}\text { Pre- } \\
\text { adminis- } \\
\text { tration }\end{array}$} & \multicolumn{8}{|c|}{ Time after administration (min) } \\
\hline & & & 15 & 30 & 45 & 60 & 75 & 90 & 105 & 120 \\
\hline Model & - & 0 & $-3.7 \pm 3.9$ & $-3.2 \pm 3.7$ & $1.9 \pm 4.6$ & $-4.3 \pm 4.9$ & $-2.7 \pm 3.0$ & $-0.3 \pm 3.7$ & $1.0 \pm 5.7$ & $3.9 \pm 3.6$ \\
\hline rhPro-UK & 2.5 & 0 & $-2.7 \pm 4.4$ & $1.4 \pm 3.8$ & $0.0 \pm 3.1$ & $6.1 \pm 4.1$ & $11.3 \pm 4.5^{\mathrm{a}}$ & $10.8 \pm 3.0$ & $15.5 \pm 5.9$ & $18.8 \pm 4.9^{\mathrm{a}}$ \\
\hline rhPro-UK & 5 & 0 & $1.2 \pm 5.2^{\mathrm{d}}$ & $10.2 \pm 5.5^{\mathrm{a}, \mathrm{d}}$ & $16.2 \pm 4.2^{\mathrm{a}, \mathrm{d}}$ & $24.7 \pm 4.9^{\mathrm{c}, \mathrm{d}}$ & $28.5 \pm 6.2^{\mathrm{c}, \mathrm{d}}$ & $27.1 \pm 6.7^{\mathrm{c}, \mathrm{d}}$ & $29.3 \pm 7.1^{\mathrm{b}, \mathrm{d}}$ & $29.9 \pm 7.1^{\mathrm{b}, \mathrm{c}}$ \\
\hline rhPro-UK & 10 & & $1.1 \pm 7.4$ & $7.4 \pm 1.6$ & $18.3 \pm 3.3^{\mathrm{a}}$ & $26.9 \pm 5.5^{\mathrm{c}}$ & $30.1 \pm 6.6^{c}$ & $37.3 \pm 6.4^{c}$ & $36.5 \pm 8.2^{\mathrm{c}}$ & $49.0 \pm 6.1^{\mathrm{c}}$ \\
\hline UK & 5 & 0 & $4.8 \pm 2.5$ & $7.6 \pm 3.4$ & $9.4 \pm 3.8$ & $13.4 \pm 5.5^{\mathrm{a}}$ & $17.0 \pm 4.3^{\mathrm{b}}$ & $17.1 \pm 5.2^{\mathrm{a}}$ & $24.4 \pm 5.9^{\mathrm{a}}$ & $22.6 \pm 6.5^{\mathrm{a}}$ \\
\hline rt-PA & $\begin{array}{c}4.5 \\
\mathrm{mg} / \mathrm{kg}\end{array}$ & 0 & $6.3 \pm 3.9$ & $13.3 \pm 4.5^{\mathrm{b}}$ & $18.4 \pm 7.9^{\mathrm{a}}$ & $24.8 \pm 7.8^{c}$ & $27.0 \pm 7.9^{c}$ & $25.7 \pm 7.5^{\mathrm{b}}$ & $29.8 \pm 7.6^{b}$ & $36.6 \pm 8.4^{c}$ \\
\hline
\end{tabular}

${ }^{\mathrm{a}} \mathrm{P}<0.05,{ }^{\mathrm{b}} \mathrm{P}<0.01$ and ${ }^{\mathrm{c}} \mathrm{P}<0.001$ vs. model group; ${ }^{\mathrm{d}} \mathrm{P}>0.05,5 \times 10^{4} \mathrm{U} / \mathrm{kg}$ rhPro-UK vs. UK. rhPro-UK, recombinant human prourokinase; UK, urokinase; rt-PA, recombinant human tissue plasminogen activator.

rate compared with $5 \times 10^{4} \mathrm{U} / \mathrm{kg}$ UK (36.8 vs. $28.3 \%$, 29.9 vs. $22.6 \%$ and 24.1 vs. $13.2 \%$; Table I and Fig. $1 \mathrm{~A}-\mathrm{C}$ ).

Effect of rhPro-UK on thrombolysis time. At the $3 \mathrm{~h}$ therapeutic time window, the thrombolysis rate of $2.5 \times 10^{4} \mathrm{U} / \mathrm{kg}$ rhPro-UK significantly increased at 75, 105 and $120 \mathrm{~min}$, at $60-120 \mathrm{~min}$ for $5 \times 10^{4} \mathrm{U} / \mathrm{kg}$ rhPro-UK and at $15-120 \mathrm{~min}$ for $10 \times 10^{4} \mathrm{U} / \mathrm{kg}$ rhPro-UK, respectively. In addition, UK and rt-PA increased the thrombolysis rate. The thrombolysis times of rhPro-UK $\left(2.5 \mathrm{x}, 5 \mathrm{x}\right.$ and $\left.10 \times 10^{4}\right)$ were $117.0,105.0$ and $77.5 \mathrm{~min}$, respectively and the thrombolysis times for UK and rt-PA were 109.0 and $97.5 \mathrm{~min}$, respectively.

At the $4.5 \mathrm{~h}$ therapeutic time window, the thrombolysis rate of $2.5 \times 10^{4} \mathrm{U} / \mathrm{kg}$ rhPro-UK significantly increased at 75 and $120 \mathrm{~min}$, at $30-120 \mathrm{~min}$ for $5 \times 10^{4} \mathrm{U} / \mathrm{kg}$ rhPro-UK and at 45-120 $\mathrm{min}$ for $10 \times 10^{4} \mathrm{U} / \mathrm{kg}$. Furthermore, UK and rt-PA increased the thrombolysis rate. The thrombolysis times of rhPro-UK $\left(2.5 \mathrm{x}, 5 \mathrm{x}\right.$ and $\left.10 \times 10^{4}\right)$ were $119.5,111.0$ and $105.5 \mathrm{~min}$, and were 111.5 and $101.5 \mathrm{~min}$ for UK and rt-PA, respectively.
At the $6 \mathrm{~h}$ therapeutic time window, the thrombolysis rate of $2.5 \times 10^{4} \mathrm{U} / \mathrm{kg}$ rhPro-UK significantly increased at $75-120 \mathrm{~min}$, at 30 and $75-120 \mathrm{~min}$ for $5 \times 10^{4} \mathrm{U} / \mathrm{kg}$ rhPro-UK, and at 30-120 $\mathrm{min}$ for $10 \times 10^{4} \mathrm{U} / \mathrm{kg}$ rhPro-UK. In addition, UK and rt-PA increased the thrombolysis rate. The thrombolysis time of rhPro-UK $\left(2.5 \mathrm{x}, 5 \mathrm{x}\right.$ and $\left.10 \times 10^{4}\right)$ were $113.0,111.0$ and 103.5, respectively, and the thrombolysis rates of UK and rt-PA were 113.0 and $107.0 \mathrm{~min}$ (Tables II-IV).

Effect of rhPro-UK on bleeding. In the different therapeutic time windows, no significant difference $(\mathrm{P}>0.05)$ was identified between rhPro-UK $\left(2.5 \mathrm{x}, 5 \mathrm{x}\right.$ and $10 \times 10^{4} \mathrm{U} /$ $\mathrm{kg}$ ) on hemorrhage type and number compared with the model group. At 3, 4.5 and $6 \mathrm{~h}$, respectively, rhPro-UK $\left(5 \times 10^{4} \mathrm{U} / \mathrm{kg}\right)$ treatment exhibited similar hemorrhage numbers when compared with UK treatment (20 vs. $30 \%$, 20 vs. $30 \%$ and 30 vs. $40 \%$ ), and the hemorrhage size also slightly decreased (1.7 vs. 3.7, 2.2 vs. 4.4 and 2.5 vs. 4.1; Table V and Fig. 2A-C). 
Table IV. Effect of rhPro-UK on thrombolysis rate (\%) and thrombolysistime in thromboembolic rabbit models at the $6 \mathrm{~h}$ therapeutic window (means \pm standard error; $n=10$ ).

\begin{tabular}{|c|c|c|c|c|c|c|c|c|c|c|}
\hline \multirow[b]{2}{*}{ Group } & \multirow{2}{*}{$\begin{array}{l}\text { Dose } \\
\left(\mathrm{x} 10^{4}\right. \\
\mathrm{U} / \mathrm{kg})\end{array}$} & \multirow{2}{*}{$\begin{array}{l}\text { Pre- } \\
\text { admini- } \\
\text { stration }\end{array}$} & \multicolumn{8}{|c|}{ Time after administration (min) } \\
\hline & & & 15 & 30 & 45 & 60 & 75 & 90 & 105 & 120 \\
\hline Model & - & 0 & $-4.6 \pm 3.3$ & $0.1 \pm 1.5$ & $0.4 \pm 3.2$ & $2.0 \pm 4.5$ & $-2.8 \pm 4.4$ & $-3.8 \pm 4.2$ & $-10.9 \pm 5.5$ & $-6.2 \pm 5.4$ \\
\hline rhPro-UK & 2.5 & 0 & $1.7 \pm 2.7$ & $3.4 \pm 3.7$ & $10.9 \pm 5.2$ & $16.8 \pm 4.0$ & $15.9 \pm 7.2^{\mathrm{a}}$ & $17.7 \pm 5.5^{\mathrm{b}}$ & $14.7 \pm 4.2^{\mathrm{b}}$ & $14.7 \pm 8.7^{\mathrm{a}}$ \\
\hline rhPro-UK & 5 & 0 & $1.9 \pm 3.2^{\mathrm{d}}$ & $9.0 \pm 2.7^{\mathrm{a}, \mathrm{d}}$ & $10.1 \pm 3.4^{\mathrm{d}}$ & $11.5 \pm 5.8^{\mathrm{d}}$ & $18.7 \pm 6.0^{\mathrm{b}, \mathrm{d}}$ & $18.0 \pm 5.8^{\mathrm{b}, \mathrm{d}}$ & $12.7 \pm 7.9^{\mathrm{b}, \mathrm{d}}$ & $24.1 \pm 6.6^{\mathrm{b}, \mathrm{d}}$ \\
\hline rhPro-UK & 10 & 0 & $0.2 \pm 4.3$ & $8.9 \pm 3.7^{\mathrm{a}}$ & $15.2 \pm 3.5^{\mathrm{b}}$ & $20.9 \pm 5.7^{\mathrm{a}}$ & $24.9 \pm 6.0^{\mathrm{c}}$ & $27.3 \pm 7.0^{\mathrm{c}}$ & $30.1 \pm 7.2^{\mathrm{c}}$ & $35.7 \pm 6.8^{c}$ \\
\hline UK & 5 & 0 & $4.5 \pm 3.8$ & $3.1 \pm 2.7$ & $1.7 \pm 3.2$ & $2.7 \pm 3.2$ & $8.0 \pm 3.5$ & $6.6 \pm 2.4$ & $5.5 \pm 1.9$ & $13.2 \pm 3.1^{\mathrm{a}}$ \\
\hline rt-PA & $\begin{array}{c}4.5 \\
\mathrm{mg} / \mathrm{kg}\end{array}$ & 0 & $3.7 \pm 3.2$ & $7.6 \pm 3.0$ & $9.0 \pm 3.6$ & $16.2 \pm 7.7$ & $28.3 \pm 7.8^{c}$ & $30.3 \pm 8.9^{c}$ & $28.2 \pm 7.4^{\mathrm{c}}$ & $30.7 \pm 7.5^{c}$ \\
\hline
\end{tabular}

${ }^{\mathrm{a}} \mathrm{P}<0.05,{ }^{\mathrm{b}} \mathrm{P}<0.01$ and ${ }^{\mathrm{c}} \mathrm{P}<0.001$ vs. model group; ${ }^{\mathrm{d}} \mathrm{P}>0.055 \times 10^{4} \mathrm{U} / \mathrm{kg}$ rhPro-UK vs. UK. rhPro-UK, recombinant human prourokinase; UK, urokinase; rt-PA, recombinant human tissue plasminogen activator.

Table V. Effect of rhPro-UK on hemorrhage in thromboembolic rabbit models $(n=10)$.

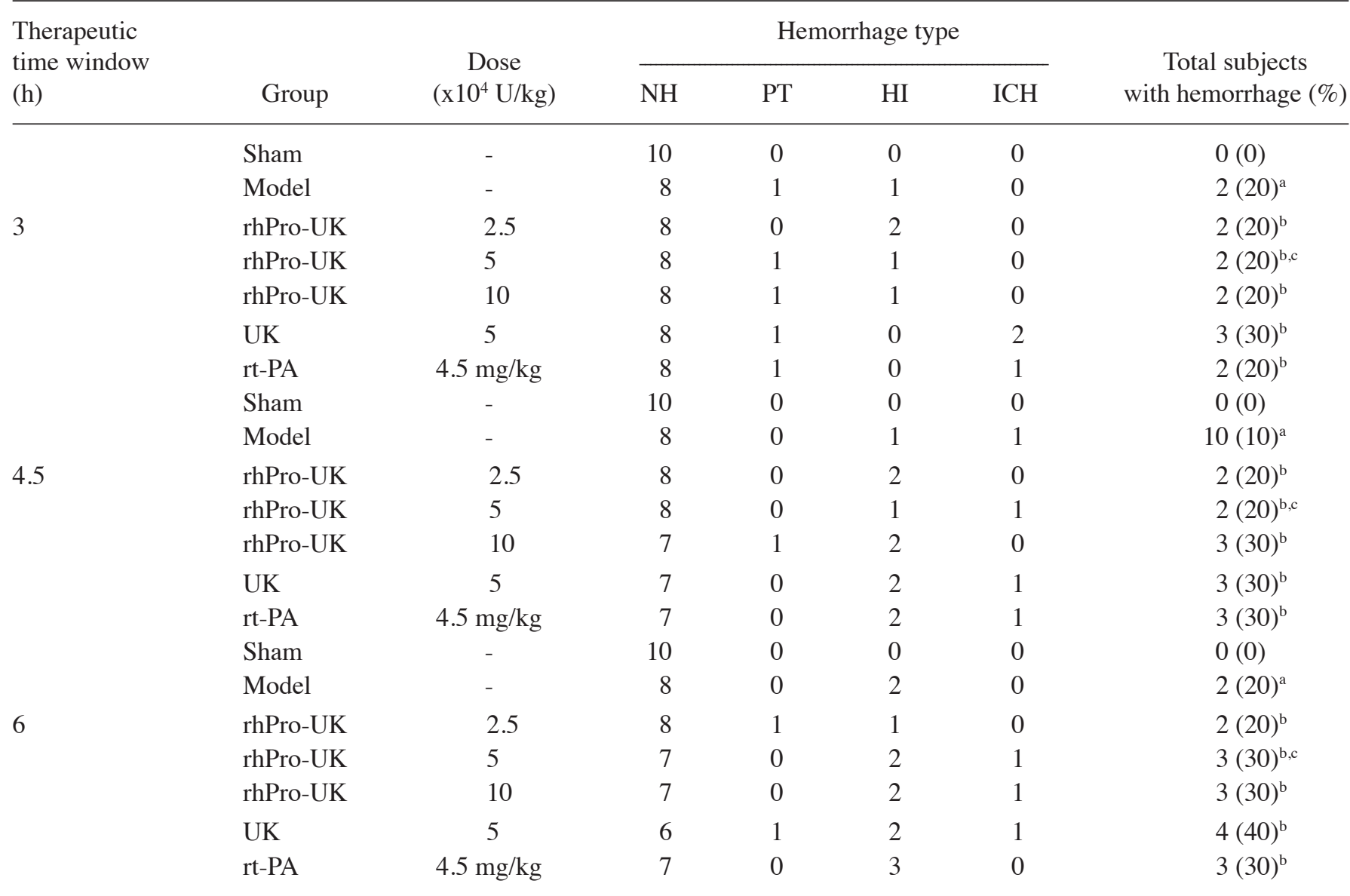

${ }^{\mathrm{a}} \mathrm{P}>0.05$ vs. sham; ${ }^{\mathrm{b}} \mathrm{P}>0.05$ vs. model group; ${ }^{\mathrm{c}} \mathrm{P}>0.055 \mathrm{x} 10^{4} \mathrm{U} / \mathrm{kg}$ rhPro-UK vs. UK. rhPro-UK, recombinant human prourokinase; UK, urokinase; rt-PA, recombinant human tissue plasminogen activator; NH, no hemorrhage; PT, punctate hemorrhage; HI, hemorrhagic infarct; ICH, intracerebral hemorrhage.

Effect of rhPro-UK on blood coagulation factor. Compared with the model group, no significant difference $(\mathrm{P}>0.05)$ was identified between rhPro-UK $\left(2.5 \mathrm{x}, 5 \mathrm{x}\right.$ and $\left.10 \times 10^{4} \mathrm{U} / \mathrm{kg}\right)$ on PT, TT, APTT and FIB for the different time windows. UK treat- ment extended TT and APTT, and reduced FIB, furthermore rt-PA prolonged APTT and reduced FIB slightly. rhPro-UK $\left(5 \times 10^{4} \mathrm{U} / \mathrm{kg}\right)$ exerted lighter effects on TT, APTT and FIB when compared with UK treatment (Table VI). 

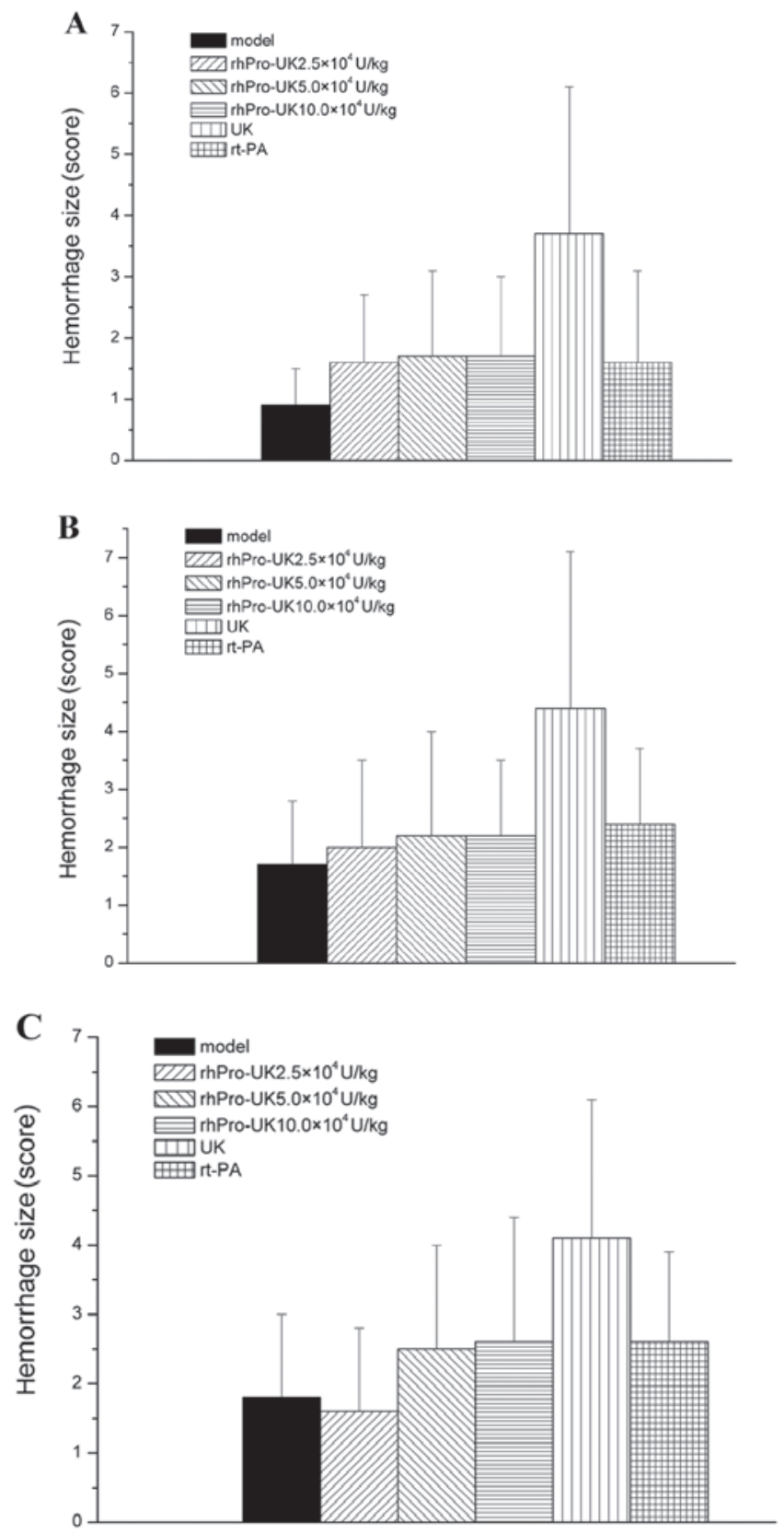

Figure 2. Effect of rhPro-UK on hemorrhage size. Thrombolysis rate was measured at (A) 3, (B) 4.5 and (C) 6 h therapeutic time windows. Data are presented as means \pm standard error $(\mathrm{n}=10)$. rhPro-UK groups compared with model, $\mathrm{P}>0.05$; rhPro-UK $5 \times 10^{4} \mathrm{U} / \mathrm{kg}$ compared with $\mathrm{UK}, \mathrm{P}>0.05$. rhPro-UK, recombinant human prourokinase; UK, urokinase; rt-PA, recombinant human tissue plasminogen activator.

Effect of rhPro-UK on $\alpha_{2}-A P$. At the $3 \mathrm{~h}$ therapeutic time window, rhPro-UK $\left(2.5 \mathrm{x}, 5 \mathrm{x}\right.$ and $\left.10 \times 10^{4} \mathrm{U} / \mathrm{kg}\right)$ reduced $\alpha_{2}-\mathrm{AP}$ by $5.3 \%(\mathrm{P}>0.05), 5.3 \%(\mathrm{P}>0.05)$ and $18.1 \%(\mathrm{P}<0.05)$, respectively. In addition, $\mathrm{UK}$ and rt-PA reduced $\alpha_{2}$-AP by $29.2 \%(\mathrm{P}<0.01)$ and $22.7 \%(\mathrm{P}<0.05)$, respectively. Compared with UK, $5 \times 10^{4} \mathrm{U} / \mathrm{kg}$ rhPro-UK exerts a smaller influence on $\alpha_{2}$-AP (9.7 vs. $\left.29.2 \%\right)$.

At the $4.5 \mathrm{~h}$ therapeutic time window, rhPro-UK $(2.5 \mathrm{x}$, $5 \mathrm{x}$ and $\left.10 \times 10^{4} \mathrm{U} / \mathrm{kg}\right)$ reduced $\alpha_{2}$-AP by $2.4 \%(\mathrm{P}>0.05)$, $6.5 \%(\mathrm{P}>0.05)$ and $17.8 \%(\mathrm{P}<0.05)$. Furthermore, $\mathrm{UK}$ and rt-PA reduced $\alpha_{2}$-AP by $25.3 \%(\mathrm{P}<0.01)$ and $19.8 \%(\mathrm{P}<0.05)$, respectively. rhPro-UK $\left(5 \times 10^{4} \mathrm{U} / \mathrm{kg}\right)$ exerts a smaller influence on $\alpha_{2}$-AP when compared with UK(6.5 vs. $\left.25.3 \%\right)$.
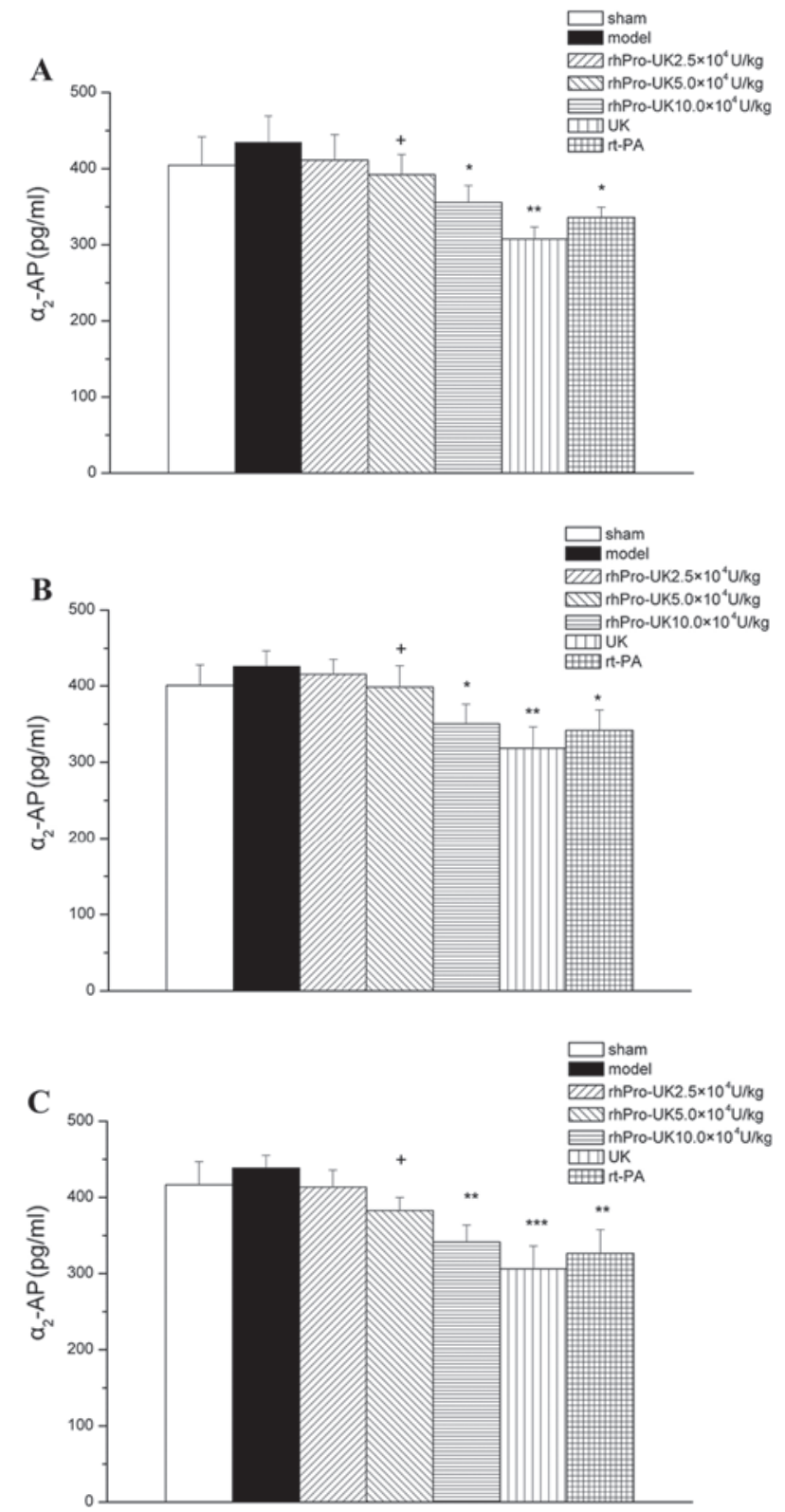

Figure 3. Effect of rhPro-UK on $\alpha_{2}$-AP production in thromboembolic rabbits. $\alpha_{2}$-AP level was measured at (A) 3, (B) 4.5 and (C) $6 \mathrm{~h}$ therapeutic time windows. Data are presented as means \pm standard error $(n=10) .{ }^{*} \mathrm{P}<0.05$, ${ }^{* *} \mathrm{P}<0.01$ and ${ }^{* * * *} \mathrm{P}<0.001$ vs. model group; ${ }^{+} \mathrm{P}<0.05$ vs. UK. Model compared with sham, $\mathrm{P}>0.05$. rhPro-UK, recombinant human prourokinase; UK, urokinase; rt-PA, recombinant human tissue plasminogen activator; $\alpha_{2}$-AP, $\alpha_{2}$-antiplasmin.

At the $6 \mathrm{~h}$ therapeutic time window, rhPro-UK $(2.5 \mathrm{x}, 5 \mathrm{x}$ and $\left.10 \times 10^{4} \mathrm{U} / \mathrm{kg}\right)$ reduced $\alpha_{2}$-AP by $5.7 \%(\mathrm{P}>0.05), 12.7 \%$ $(\mathrm{P}>0.05)$ and $22.2 \%(\mathrm{P}<0.01)$. In addition, $\mathrm{UK}$ and rt-PA reduced $\alpha_{2}$-AP by $30.2 \%(\mathrm{P}<0.001)$ and $25.6 \%(\mathrm{P}<0.01)$. Compared with UK, $5 \times 10^{4} \mathrm{U} / \mathrm{kg}$ rhPro-UK exerts a smaller influence on $\alpha_{2}$-AP when compared with UK (12.7 vs. 30.2\%; Fig. 3A-C).

\section{Discussion}

Stroke is the second leading cause of mortality worldwide and the number one cause of disability in the USA (17). IV tPA remains the only drug that has been approved by the United 
Table VI. Effect of rhPro-UK on blood coagulation factors in thromboembolic rabbit models (means \pm standard error; $\mathrm{n}=10$ ).

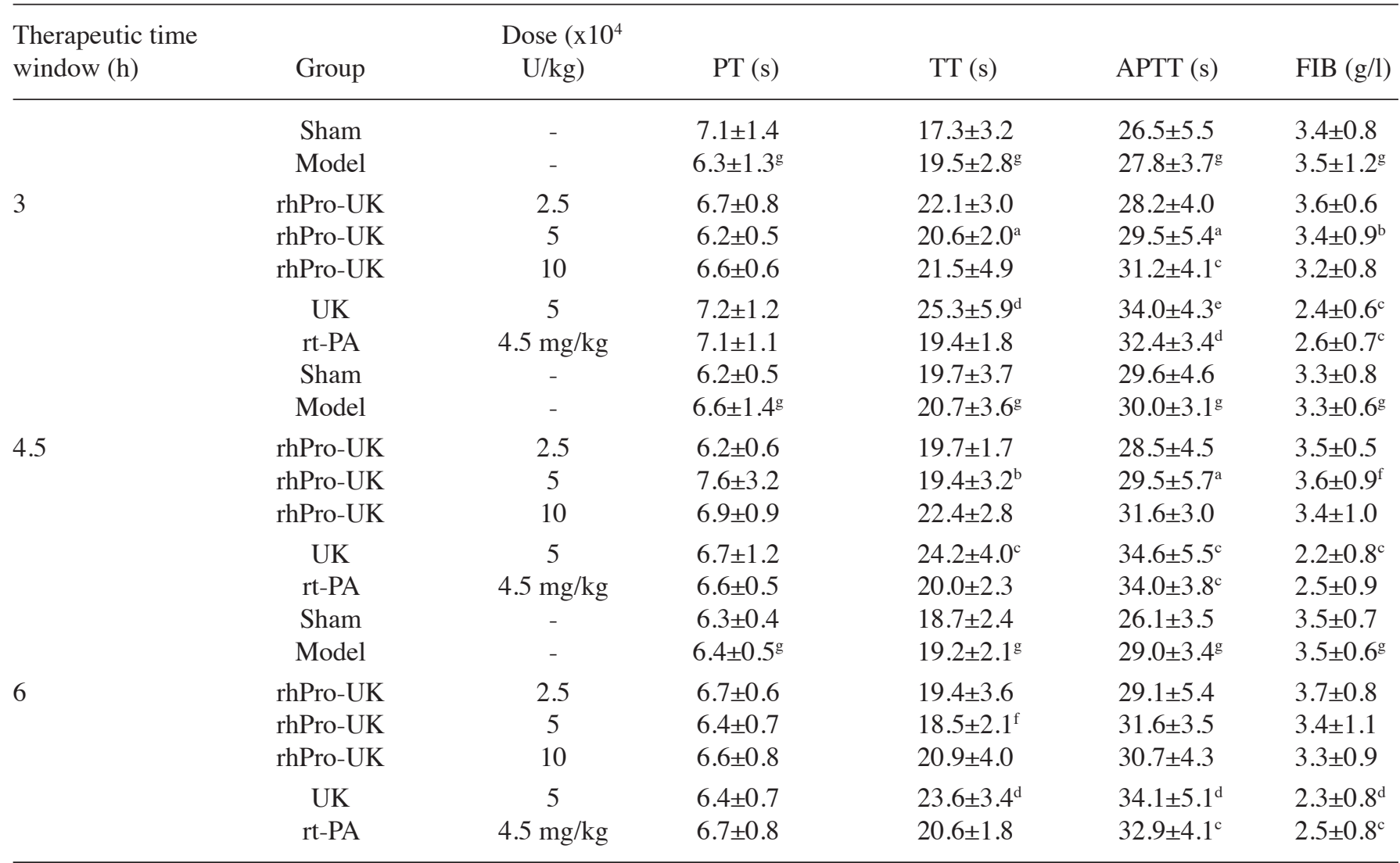

${ }^{\mathrm{a}} \mathrm{P}<0.05$ and ${ }^{\mathrm{b}} \mathrm{P}<0.01$ vs. $\mathrm{UK} ;{ }^{\mathrm{c}} \mathrm{P}<0.05,{ }^{\mathrm{d}} \mathrm{P}<0.01$ and ${ }^{\mathrm{e}} \mathrm{P}<0.001$ vs. model; ${ }^{\mathrm{f}} \mathrm{P}<0.001$ vs. UK; ${ }^{\mathrm{g}} \mathrm{P}>0.05$, model vs. sham. rhPro-UK, recombinant human prourokinase; UK, urokinase; rt-PA, recombinant human tissue plasminogen activator; APTT, activated partial thromboplastin time; PT, prothrombin time; TT, thrombin time; FIB, fibrinogenemia.

States Food and Drug Administration for its treatment (18). However, the perception of marginal utility, high risk of intracerebral bleeding, and/or high liability associated with its administration discourage its administration (19), although the American Heart Association has deemed it an acceptable alternative therapy and many stroke centers offer it to patients within $6 \mathrm{~h}$ of a major acute stroke (20). These limitations reflect the requirement for more effective thrombolic drugs. rhPro-UK has more potent efficacy and fewer adverse reactions in comparison with other thrombolytics due to the fibrin-selective clot lysis. The rhPro-UK in the present study was from Tasly Pharmaceutical Co., Ltd., generated from Chinese hamster ovary cell expression using a genetic engineering method, and is typically used to treat acute myocardial infarction (21). The present study evaluated IV thrombolysis with rhPro-UK in rabbit acute cerebral infarction at 3, 4.5 and $6 \mathrm{~h}$ therapeutic time windows.

The results confirmed that the thrombolysis rate and patency rate (recanalization rate) increased as the time window shortened. At 3, 4.5 and $6 \mathrm{~h}$ therapeutic time windows, the thrombolysis rate of $5 \times 10^{4} \mathrm{U} / \mathrm{kg}$ rhProUK was $36.8,29.9$ and $24.1 \%$, respectively and the patency rate was 40,30 and $30 \%$. The thrombolysis rate of $10 \times 10^{4} \mathrm{U} / \mathrm{kg}$ rhPro-UK was $55.0,49.0$ and $35.7 \%$ and the patency rate was $70,50,40 \%$ at $3,4.5$ and $6 \mathrm{~h}$ therapeutic time windows, respectively. rhPro-UK treatment increased the thrombolysis rate slightly when compared with UK (36.8 vs. $28.3 \%, 29.9$ vs. $22.6 \%$ and 24.1 vs. $13.2 \%$ ). Consistent with the present study, del Zoppo et al (3) reported a phase II randomized trial of rhPro-UK by direct arterial delivery in acute middle cerebral artery stroke, local IV rhPro-UK infusion at $5.5 \mathrm{~h}$ from symptom onset was associated with superior recanalization in acute thrombotic/ thromboembolic stroke when compared with a placebo. In addition, Tirschwell et al (22) reported a PROACT II trial including 180 patients with acute ischemic stroke, despite an increased frequency of early symptomatic intracranial hemorrhage, treatment with Pro-UK within $6 \mathrm{~h}$ of the onset of acute ischemic stroke caused by middle cerebral artery occlusion significantly improved the clinical outcome at 90 days.

In addition, it was found that rhPro-UK $(2.5 \mathrm{x}, 5 \mathrm{x}$ and $10 \times 10^{4} \mathrm{U} / \mathrm{kg}$ ) did not increase bleeding compared with the model group $(\mathrm{P}>0.05)$, and the hemorrhage size of the $5 \times 10^{4} \mathrm{U} / \mathrm{kg}$ rhPro-UK group was slightly decreased compared with the UK treatment group at different time points. rhPro-UK $\left(5 \times 10^{4} \mathrm{U} / \mathrm{kg}\right)$ had less of an influence on PT, TT, APTT, FIB and $\alpha_{2}$-APwhen compared with UK. This finding is comparable to a study by Zhang et al (23), where rhPro-UK did not effect FIB, PA or $\alpha_{2}$-AP, and the effect on bleeding time, clotting time and bleeding quantity per unit time was less than those of UK.

Plasmin is an enzyme that participates in fibrinolysis. $\alpha_{2}$-AP is a serine protease inhibitor responsible for inactivating 
plasmin. Its rapid reaction with plasmin results in the formation of an inactive complex (plasmin- $\alpha_{2}$-AP complex; PAP), which is composed of one molecule of each component. Therefore, the method that was used for measuring $\alpha_{2}$-AP in the present study only indirectly reflects the actual fibrinolytic activity, and thus presents a limitation of this study. Determination of PAP may be more appropriate in future studies.

In conclusion, IV rhPro-UK exerted therapeutic effects on thromboembolic stroke rabbit models within a $6 \mathrm{~h}$ time frame, influencing thrombolysis and recanalization (patency rate) with reduced risk of cerebral hemorrhage.

\section{References}

1. Furlan A, Higashida R, Wechsler L, Gent M, Rowley H, Kase C, Pessin M, Ahuja A, Callahan F, Clark WM, et al: Intra-arterial prourokinase for acute ischemic stroke. The PROACT II study: A randomized controlled trial. Prolyse in acute cerebral thromboembolism. JAMA 282: 2003-2011, 1999.

2. Gurewich V, Pannell R, Louie S, Kelley P, Suddith RL and Greenlee R: Effective and fibrin-specific clot lysis by a zymogen precursor form of urokinase (pro-urokinase). A study in vitro and in two animal species. J Clin Invest 73: 1731-1739, 1984.

3. del Zoppo GJ, Higashida RT, Furlan AJ, Pessin MS, Rowley HA and Gent M: PROACT: A phase II randomized trial of recombinant pro-urokinase by direct arterial delivery in acute middle cerebral artery stroke. PROACT investigators. Prolyse in acute cerebral thromboembolism. Stroke 29: 4-11, 1998.

4. Moia M, Mannucci PM, Pini M, Prandoni P and Gurewich V: A pilot study of pro-urokinase in the treatment of deep vein thrombosis. Thromb Haemost 72: 430-433, 1994.

5. Ning RX, Wang R and Cui XY: A new thrombolytic drug: Recombinant human prourokinase. Zhongguo Xin Yao Zazhi 17: 430-432, 2008 (In Chinese).

6. Liu Y and Wang L: Efficacy and safety of thrombolytic therapy with recombinant human prourokinase for acute myocardial infarction in 50 cases. Chin Pharm J 15: 76-78, 2015 (In Chinese).

7. Thomas GR, Thibodeaux H, Bennett WF, Refino CJ, Badillo JM, Errett CJ and Zivin JA: Optimized thrombolysis of cerebral clots with tissue-type plasminogen activator in a rabbit model of embolic stroke. J Pharmacol Exp Ther 264: 67-73, 1993.

8. Hao CH, Xu XW, Ma YZ, Zhang R, Sun SY, Wang WT, et al: Application of ${ }^{99} \mathrm{Tc}^{\mathrm{m}}$ tracer technique in rabbit cerebral thromboembolic stroke. Yaowu Pingjia Yanjiu 40: 648-651, 2017 (In Chinese).

9. Zhang L, Zhang RL, Jiang Q, Ding G, Chopp M and Zhang ZG: Focal embolic cerebral ischemia in the rat. Nat Protoc 10: 539-547, 2015.

10. Feng L, Liu J, Chen J, Pan L and Feng G: Establishing a model of middle cerebral artery occlusion in rabbits using endovascular interventional techniques. Exp Ther Med 6: 947-952, 2013.
11. Zhang Z, Zhang RL, Jiang Q, Raman SB, Cantwell L and Chopp M: A new rat model of thrombotic focal cerebral ischemia. J Cereb Blood Flow Metab 17: 123-135, 1997.

12. Yu B, Tong $\mathrm{J}$ and Li HF: Thrombolytic efficacy of rt-PA in embolic stroke of rabbits by ${ }^{99} \mathrm{Tc}^{\mathrm{m}}$ trace. J Isot 18: 160-163, 2005.

13. Thomas GR, Thibodeaux H, Errett CJ, Badillo JM, Keyt BA and Refino CJ, Zivin JA, Bennett WF, et al. A long-half-life and fibrin-specific form of tissue plasminogen activator in rabbit models of embolic stroke and peripheral bleeding. Stroke 25: 2072-2078, 1994.

14. Bovill EG, Terrin ML, Stump DC, Berke AD, Frederick M, Collen D, Feit F, Gore JM, Hillis LD, Lambrew CT, et al: Hemorrhagic events during therapy with recombinant tissue-type plasminogen activator, heparin, and aspirin for acute myocardial infarction. Results of the thrombolysis in myocardial infarction (TIMI), Phase II trial. Ann Intern Med 115: 256-265, 1991.

15. Clark WM, Madden KP, Lyden PD and Zivin JA: Cerebral hemorrhagic risk of aspirin or heparin therapy with thrombolytic treatment in rabbits. Stroke 22: 872-876, 1991.

16. Chapman DF, Lyden P, Lapchak PA, Nunez S, Thibodeaux H and Zivin J: Comparison of TNK with wild-type tissue plasminogen activator in a rabbit embolic stroke model. Stroke 32: 748-752, 2001.

17. Mozaffarian D, Benjamin EJ, Go AS, Arnett DK, Blaha MJ, Cushman M, de Ferranti S, Després JP, Fullerton HJ, Howard VJ, et al: Heart disease and stroke statistics-2015 update: A report from the American heart association. Circulation 131: 29-322, 2015.

18. Amar AP, Griffin JH and Zlokovic BV: Combined neurothrombectomy or thrombolysis with adjunctive delivery of 3K3A-activated protein $\mathrm{C}$ in acute ischemic stroke. Front Cell Neurosci 9: 344, 2015.

19. SoRelle and Ruth MP: Breaking news: The 'biggest, baddest' controversy in EM. Emerg Med News 35, 26-27, 2013.

20. Furlan AJ and Abou-Chebl A: The role of recombinant pro-urokinase (r-pro-UK) and intra-arterial thrombolysis in acute ischaemic stroke: The PROACT trials. Prolyse in acute cerebral thromboembolism. Curr Med Res Opin 18: 44-47, 2002.

21. Prourokinase Clinical Trial Group: Li T, Xiao Ch, Liu R and Liu L: Multicenter phase III study of recombinant prourokinase for acute myocardial infarction with ST-segment evaluation. J Med Res 42: 26-31, 2013.

22. Tirschwell DL, Coplin WM, Becker KJ, Vogelzang P, Eskridge J, Haynor D, Cohen W, Newell D, Winn HR and Longstreth WT Jr: Intra-arterial urokinase for acute ischemic stroke: Factors associated with complications. Neurology 57: 1100-1103, 2001.

23. Zhang ZG, Xiao CZ, Hu XW, Xu ZP, Liu JX, Yang SJ, Ren JP, Liao MY, Shi XC, Wu BA: Investigation of pharmacodynamics, pharmacology, and toxicology of domestic human prourokinase. Sci Sin Vitae 41: 1024-1029, 2011. 